\title{
FIELD ERRORS IN HYBRID INSERTION DEVICES*
}

\author{
Ross D. Schlueter \\ Advanced Light Source \\ Accelerator and Fusion Research Division \\ Lawrence Berkeley Laboratory \\ Berkeley, CA 94720, USA
}

February 3, 1995

Halbach Symposium on Magnet Technology

Lawrence Berkeley Laboratory

*This work was supported by the Director, Office of Energy Research, Office of Basic Energy Sciences, Materials Sciences Division, of the U.S. Department of Energy, under Contract No. DE-AC03-76SF00098. 


\section{DISCLAIMER}

This report was prepared as an account of work sponsored by an agency of the United States Government. Neither the United States Government nor any agency thereof, nor any of their employees, make any warranty, express or implied, or assumes any legal liability or responsibility for the accuracy, completeness, or usefulness of any information, apparatus, product, or process disclosed, or represents that its use would not infringe privately owned rights. Reference herein to any specific commercial product, process, or service by trade name, trademark, manufacturer, or otherwise does not necessarily constitute or imply its endorsement, recommendation, or favoring by the United States Government or any agency thereof. The views and opinions of authors expressed herein do not necessarily state or reflect those of the United States Government or any agency thereof. 


\section{DISCLAIMER}

Portions of this document may be illegible in electronic image products. Images are produced from the best available original document. 


\title{
FIELD ERRORS IN HYBRID INSERTION DEVICES ${ }^{\dagger 1}$
}

\author{
Ross D. Schlueter \\ Lawrence Berkeley Laboratory \\ 1 Cyclotron Road, MS 46-161 \\ Berkeley, CA 94720, USA ${ }^{\ddagger 2}$
}

\begin{abstract}
Hybrid magnet theory as applied to the error analyses used in the design of Advanced Light Source (ALS) insertion devices is reviewed. Sources of field errors in hybrid insertion devices are discussed.
\end{abstract}

\section{Introduction}

Klaus Halbach is well known for originating and developing permanent magnet (PM) technology for accelerator magnets, including insertion devices (IDs). An equally important contribution, though perhaps less glamorous, is his pioneering work in error analyses, both their theoretical calculation and effects, applicable to these accelerator magnets, again including IDs $[1,2]$. Hybrid IDs, employing both permanent magnet material and soft iron, were pioneered at LBL and have since become popular in synchrotron sources worldwide. Recently several outside requests have been received to set forth the error analysis theory used in the design of these devices. That follows herein. Implications for resulting mechanical tolerances and permanent magnet block quality specifications are already described in the various ALS ID conceptual design reports [3-7].

Yet another immense contribution of Klaus's is his mentorship of numerous scientists and engineers and the "little-boy-excitement" he transmits to those that work with him in the field of accelerator magnet design and technology. This paper is dedicated to Klaus Halbach in appreciation for both the broad training graciously tendered and the little-boy-excitement for the subject he has infused in me.

\section{Permanent Magnets: Passive Materials with Active Terms}

In directions parallel and perpendicular to the magnetization of a permanetly magnetized material the induction $B$ is related to the field $H$ by, respectively

$$
B_{\|}=\mu_{\|} H_{\|}+B_{r} \quad \text { and } \quad B_{\perp}=\mu_{\perp} H_{\perp}
$$

where $B_{r}$ is the remanent field and $\mu$ is the differential permeability. Vectorially the $B-H$ relation may be expressed

$$
\vec{B}=\hat{\mu} \vec{H}+\vec{B}_{\tau} \quad \text { or } \quad \vec{H}=\hat{\gamma} \vec{B}-\vec{H}_{c},
$$

${ }^{1 \dagger}$ This work was supported by the Director, Office of Energy Research, Office of Basic Energy Sciences, Materials Science Div., of the U.S. Dept. of Energy under Contract No. DEAC03-76SF00098.

2ł E-mail: ross@lbl.gov, Fax: 510-486-4873. 
$\vec{B}:$ PM CURRENTS
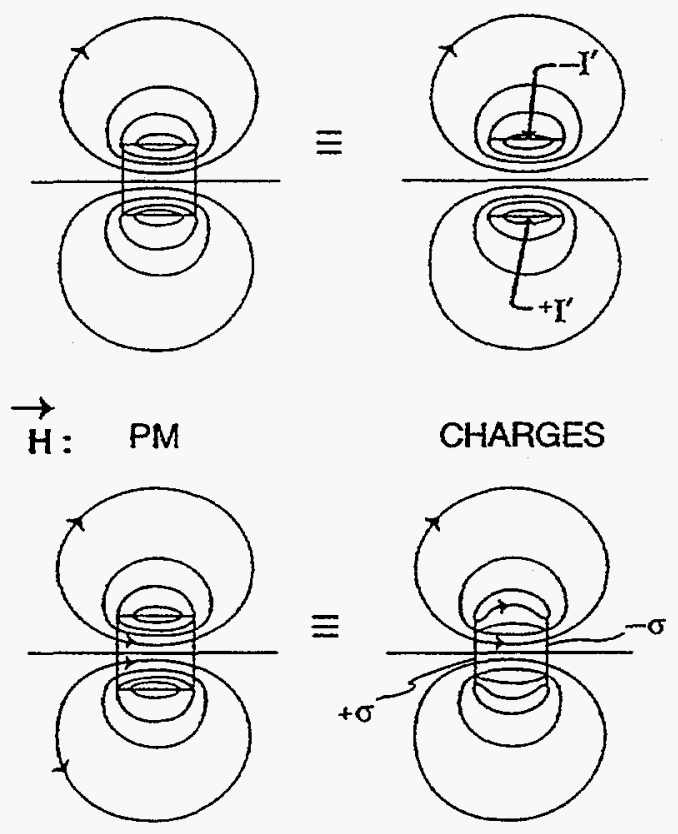

Figure 1. Plots of (a) $B$ and (b) $H$ for a homogeneously magnetized PM block in free space and for equivalent current or charge sheet models.

where $H_{c}$ is the coersive force and $\hat{\gamma} \equiv \hat{\mu}^{-1}$. In a current-free, time-independent region, it follows from Maxwell's equations $\vec{\nabla} \times \vec{H}=0$ and $\vec{\nabla} \cdot \vec{B}=0$. Thus the divergence of Eq. (2a) and the curl of Eq. (2b) yield, respectively,

$$
\begin{aligned}
& \vec{\nabla} \cdot \hat{\mu} \vec{H}=-\vec{\nabla} \cdot \vec{B}_{r} \equiv \rho_{e q} \quad \text { or } \quad \vec{\nabla} \times \hat{\gamma} \vec{B}=-\vec{\nabla} \times \vec{H}_{c} \equiv \mathrm{Jeq} \\
& \text { with } \vec{\nabla} \times \vec{H}=0 \quad \text { with } \vec{\nabla} \cdot \vec{B}=0 \text {, }
\end{aligned}
$$

where $\rho_{e q}$ and Jeq are equivalent charge and current densities, respectively. The PM material thus can be represented as a passive material of permeability $\hat{\mu}$ or $\hat{\gamma}^{-1}$ with active source terms $\rho_{e q}$ or $j_{e q}$. For a homogeneously magnetized PM block $\vec{\nabla} \cdot \vec{B}_{r}$ and. $\vec{\nabla} \times \vec{H}_{c}$ are zero everywhere except at surfaces parallel and perpendicular, respectively, to the block magnetization, giving rise to surface charge sheets $\sigma \equiv \rho_{e q} \delta= \pm B_{r}[\mathrm{G}]$ or current sheets $I^{\prime} \equiv \mathrm{j}_{e q} \delta= \pm H_{c}[\mathrm{G}]$, respectively, where $\delta$ is the infinitesimal sheet thickness. Figure $1 \mathrm{a}, \mathrm{b}$ plot $B$ and $H$, respectively for a homogeneously magnetized PM block and for either equivalent current or charge sheets, respectively. The remaining quantity, $H$ or $B$, can be obtained from the $B-H$ relation, e.g., Eq. (2). The high strength $\mathrm{Nd}-\mathrm{Fe}-\mathrm{B}, \mathrm{Sm}-\mathrm{Co}$, and ferrite PMs used in accelerator magnets exhibit permeabilities $\mu \simeq 1.04$, thus it is often convenient to model these with $\pm B_{r}$ charge sheets separated by effectively free space. 


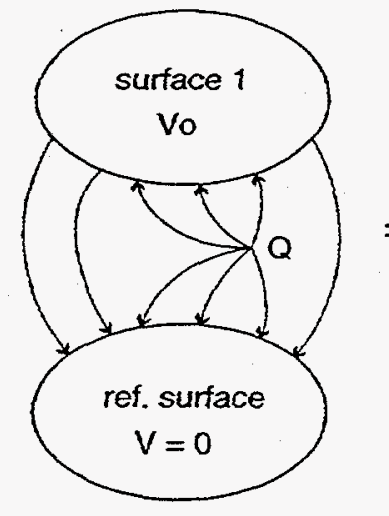

(c)

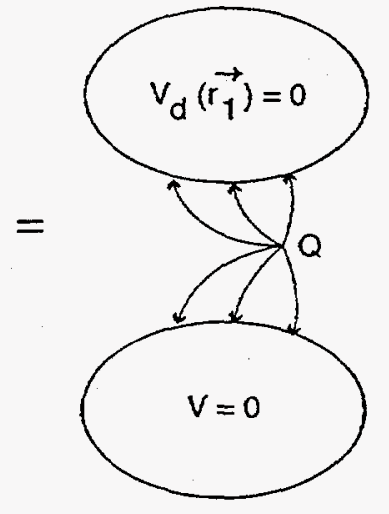

(a)

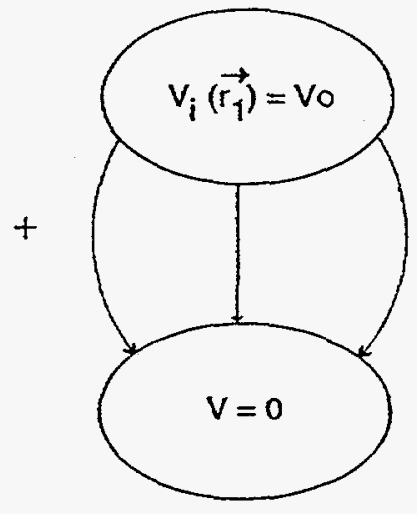

(b)

INDIRECT FIELDS

Figure 2. Hybrid solution for two infinite $\mu$ bodies in the presence of a point charge $Q$ : (c) the total solution; (a) the direct field component, (b) the indirect field component.

3 Hybrid Magnets: Zero Reluctance Volumes with Iso-Scalar Potential Field Shaping Surfaces passing Zero Net Flux

A hybrid magnet can be modeled by charge sheets at appropriate PM surfaces and by iso-scalar potential surfaces enclosing the reluctance-free soft iron. Imagine a single point charge $Q$ near two iso-scalar potential surfaces. A field solution can be constructed that satisfies Maxwell's equations in space outside the iron and has net flux $\Phi_{1}=0$ entering surface 1 on scalar potential $V_{0}$. (The other is a reference surface pegged to a scalar potential $V=0$, without loss of generality.) As shown by Fig. 2, this solution, (c), is a superposition of two solutions, (a) and (b), to Maxwell's equations outside the iron. The first solution, (a), comprises the direct flux emanating from the point charge being deposited on either of the two surfaces, both of whose surfaces are on iso-scalar potential contours $V=0$. The second solution, (b), comprises the indirect flux arising from assumed different scalar potentials of the two surfaces, in the absence of all sources (charges). In fact, the equilibrium scalar potential $V=V_{0}$ that surface 1 assumes is that required to satisfy $\int_{a_{1}} \vec{B} \cdot d \vec{a}_{1}=0$, where $a_{1}$ is the area of surface 1, i.e., no net flux enters the iron.

In the first solution, (a), the direct flux deposited on surface $1, \Phi_{1_{d}}=f Q,(0 \leq$ $f \leq 1$ ), where $f$, the fraction of $Q$ deposited on surface 1 , is readily obtained from the geometry of the second solution, (b): $f$ is merely the scalar potential at the location of the charge (in its absence) normalized with respect to the potential of surface 1, i.e., $f=V_{i}\left(\vec{r}_{Q}\right) / V_{0}$. The remainder, $(1-f) Q$ is deposited on the reference surface. That this is so is somewhat intuitive; the source in a vacuum region near two zero potential surfaces is analogous to a voltage divider. A rigorous proof is given in Appendix A.

In the second solution, (b), the indirect flux into surface $1, \Phi_{1_{i}}=-V_{0} c_{2}$, where $V_{0}$ is still unknown and where the "capacitance" $c_{2}$, is a constant of proportionality 
readily determined from the geometry alone by solving the boundary value problem, (b), with any assumed value of $V_{0}$.

Finally, in the combined solution, (c), the net flux crossing surface 1 is zero, which determines the value of the equilibrium scalar potential $V_{0}$ :

$$
\Phi_{1_{i}}+\Phi_{1_{d}}=0 \quad \Longrightarrow \quad V_{0}=Q f / c_{2} .
$$

Zero net flux across the reference surface is likewise achieved when the necessary complimentary negative point charge $-Q$ is added to the model. For a dipole then, the direct flux deposited on surface 1 is.

$$
\Phi_{1_{d}}=Q\left(V_{i}\left(\vec{r}_{Q}+\overrightarrow{\delta r}_{Q}\right)-V_{i}\left(\vec{r}_{Q}\right)\right) / V_{0}=-Q \overrightarrow{\delta r_{Q}} \cdot \vec{H}_{i}\left(\vec{r}_{Q}\right) / V_{0}
$$

and for a PM block, with $Q \overrightarrow{\delta r}_{Q}=\left|B_{r}\right| a_{P M} \overrightarrow{\delta r}_{Q}=\vec{B}_{r} d v$, the direct flux deposited on surface 1 is

$$
\Phi_{1_{d}}=-\int \vec{B}_{r} \cdot \vec{H}_{i} d v / V_{0} .
$$

The total solution to a hybrid system then, consists of solving a boundary problem and integrating over the charge distribution modeling the PM material to obtain $f$ (or equivilently $\Phi_{1_{d}}$ ) and $c_{2}$.

Applying this theory to a hybrid ID design, let surface 1 be that of a soft iron undulator pole and the reference surface be the zero scalar potential surface consisting of the ID midplane and the vertical planes midway between adjacent poles. Then $c_{2} V_{0}$ is the indirect flux leaving the pole sitting on scalar potential $V_{0}$. The design of a hybrid ID entails selecting the pole tip shape that produces the desired [indirectflux-induced] field distribution, then determining the pole scalar potential required to achieve the desired field strength, and finally designing the rest of the iron and [direct-flux-generating] PM to produce this potential. It should be noted that in a hybrid design the PM is positioned so the direct flux goes where one desires, i.e., either to/from an energized pole or from/to a non-critical portion of a zero scalar potential surface, thus leaving only indirect flux in the "business region". Detailed hybrid theory analytical calculation for accelerator magnet design (including IDs) is an interesting subject in itself, but is not treated here.

\section{Partitioning of the Capacitance $c_{2}$}

Here we are interested in the integrated error flux that the electron beam sees. It is thus incumbent on us to determine both how much direct flux and how much indirect flux crosses the midplane in the region underneath the pole-width-envelope where the field is invariant in the transverse direction. For this reason, though not for the hybrid ID design itself, it is necessary to partition the capacitance $c_{2}$, as shown below. (Partitioning of $c_{2}$ is likewise integral to analytical design of ID ends and calculation of error propagation through the ID.)

The hybrid theory introduced in the preceeding section may be rigorously extended to systems with multiple isopotential surfaces. Here we take advantage of inherent. 


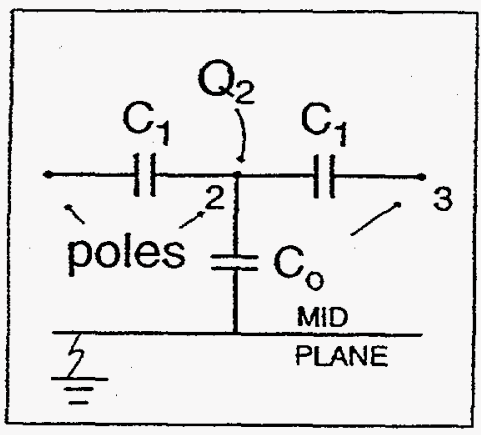

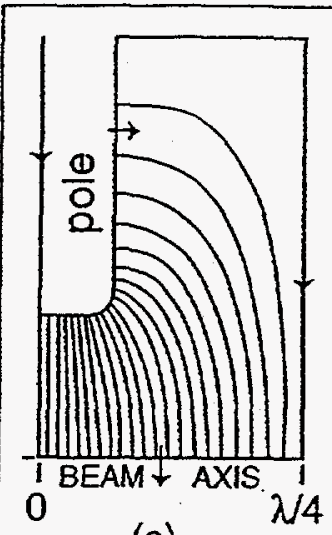

(a)

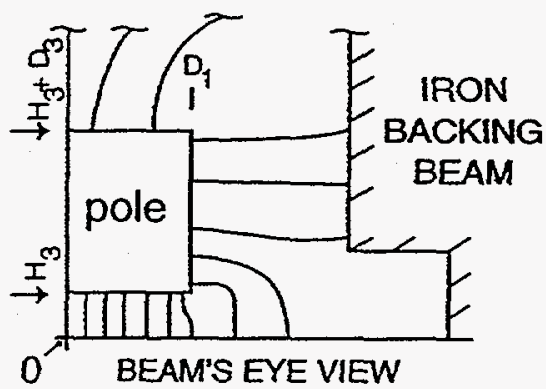

(b)

Figure 3. ID circuit model. Figure 4. (a) 2-D and (b) fringe flux to ID reference surface for determination of capacitance $c_{0}$.

ID symmetries allowing simple partitioning of the capacitance $c_{2}$. Figure 3 shows an electrical analog of an undulator where the nodes are poles, with the reference ground on $V=0$ being the ID midplane.

For,,,+-+- pole excitation, the PM-induced charge $Q_{2}$ into pole $\# 2$ on $+V_{0}$ leaves the pole as indirect tlux to either pole \#1 or pole \#3, both on potential $-V_{0}$ or to to the midplane on $V=0$. The direct flux $Q_{2}$ is then given by

$$
Q_{2}=V_{0}\left(c_{0}+4 c_{1}\right)
$$

We already know from the hybrid design, which assumes,,,+-+- pole excitation, that the direct flux entering a pole is equal to the indirect flux leaving, which in turn is proportional to the pole potential $V_{0}$, via the capacitance $c_{2}$. The $c_{2}$ then can be partitioned as follows:

$$
\Phi_{d}\left(=Q_{2}\right)=-\Phi_{i}=c_{2} V_{0} \quad \Longrightarrow \quad c_{2}=c_{0}+4 c_{1} \text {. }
$$

With $c_{2}$ known from the [analytical or numerical] solution of a boundary value problem, it remains to calculate either $c_{1}$ or $c_{0}$. Consider a fictitious,,,++++ pole excitation, where all poles lie on isopotential $V=V_{2}$. In this case there is no net flux pole-to-pole and $100 \%$ of the charge $Q$ into pole \#2 leaves the pole as indirect tlux to the midplane on $V=0$ :

$$
Q=V_{2} c_{0}
$$

Two parts contribute to $c_{0}$ as shown in Fig. $4 a, b$ : (a) The 2-D portion in the region underneath the pole-width-envelope where the field is invariant in the transverse direction is given by

$$
c_{0_{f}} / 4=[A(\lambda / 4,0)-A(0,0)] D_{1} / V_{2}
$$


(a)

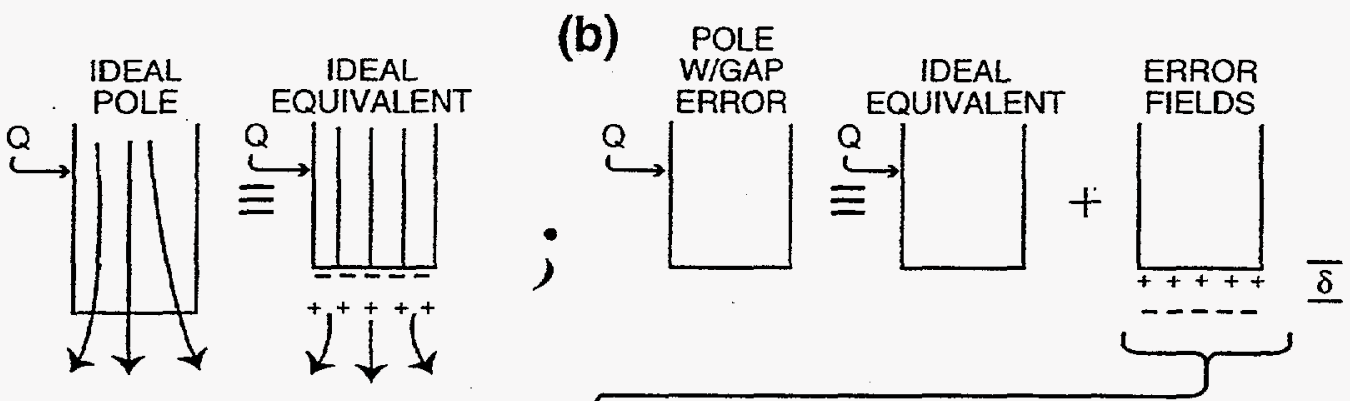

(c)

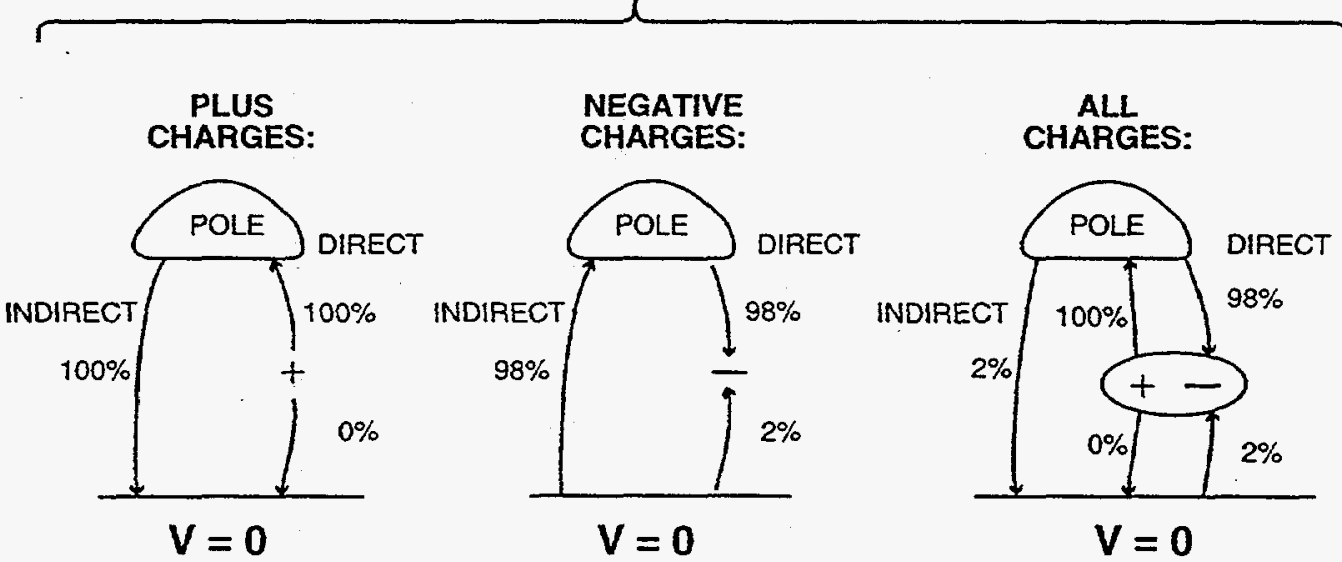

Figure 5. Modeling iron displacement with charge sheet equivalents:

(a) an ideal pole, (b) a shortened pole, (c) direct and indirect error flux from positive and negative charge sheets.

where coordinates are in the $z-y$ plane, $A$ is the 2-D magnetostatic vector potential $(\nabla \times A=B), \lambda$ is the ID period, and $D_{1}$ is the pole half-width in the transverse direction; (b) The fringe flux at the side and top surfaces of the pole is given by

$$
c_{0_{s}} / 4=\left[A\left(0, H_{3}+D_{3}\right)-A\left(D_{1}, 0\right)\right][\lambda / 4] / V_{2},
$$

where coordinates are in the $x-y$ plane, $H_{3}$ and $D_{3}$ are the half-gap and pole height, respectively. The nearby iron backing beam must be considered part the the zero scalar potential reference surface. To be rigorous one must modify the $c_{0}$ calculation to account for an excess voltage drop associated with the fact that the pole does not extend axially to $\lambda / 4$. Also one could include capacitances between non-adjacent poles. Both these are relatively minor effects.

\section{ID Error Sources and Their Effect on Integrated Field Errors}

An ideal pole energized by charge $Q$ and an energized charge sheet ideal equivalent recessed pole with charge sheets separated by the recessed amount $\delta$ yield identical flux distributions, as shown in Fig. 5a. An energized pole exhibiting a gap error thus, can be modeled by superposing to an energized ideal equivalent recessed pole an unenergized recessed pole with charge sheets of the opposite polarity, as shown in Fig. 5b. The decomposition of the error fields is shown in Fig. $5 \mathrm{c}$ for the plus charges 

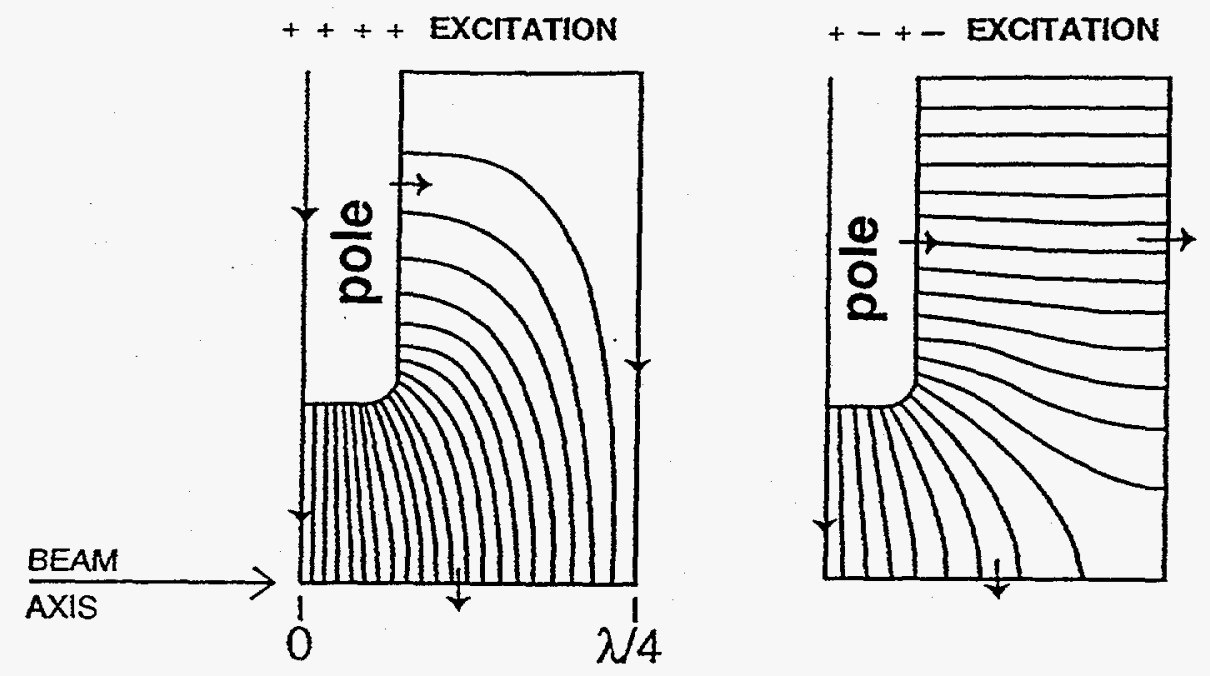

Figure 6. A quarter-period ID model with adjacent pole excitation patterns of:

(a),,,++++ , and (b),,,+-+-

and for the negative charges separately. The direct error flux going to the midplane, $Q_{0}$ is all from the negative charges. It may be calculated following the argument set forth in section 3: The magnitude of the charge density $q^{-}=B_{+-+-}$, where $B_{+-+-}$ is the field at the location $r_{q-}$ for the,,,+-+- pole excitation shown in Fig 6a. The $f$, the fraction of $q^{-}$that goes directly to the midplane, found by putting all poles on zero scalar potential and the midplane on $V_{2}$, is just $f=V_{i}\left(r_{q^{-}}\right) / V_{0}=B_{++++} \delta / V_{0}$, where $B_{++++}$is the field at the location $r_{q^{-}}$for the,,,++++ pole excitation shown in Fig $6 b$, and $\delta$ is the half-gap error. Error flux to the midplane is thus

$$
Q_{0}=\delta \int B_{+++-}(s) B_{++++}(s) d s / V_{2},
$$

where $d s$ is along the charge sheet.

From Fig. 5c, an equal amount of direct flux (100\% of the + charges and $98 \%$ of the - charges in this hypothetical example shown) but of opposite polarity $-Q_{0}$ goes to the poles. Indirect fields must deposit this flux on the midplane, but only a fraction $c_{0_{f}} /\left(c_{0_{f}}+c_{0_{s}}\right)$ is seen by the electron beam, per the argument of the preceding section. Thus the net flux that the electron beam sees is

$$
Q_{\text {beam }}=Q_{0}\left(1-\left[c_{f} /\left(c_{0_{f}}+c_{0_{s}}\right)\right]\right)=Q_{0} c_{0_{s}} /\left(c_{0_{f}}+c_{0_{s}}\right) .
$$

Various error sources are depicted in Fig. 7. Pole thickness error is modeled analogously, following the pole gap error method. Spacing between the PM and the pole is analogously modeled with a perfectly sized PM block and a superimposed PM block sliver of thickness $\delta$ and with magnetization in the opposite direction as the ideal PM block. An easy axis orientation error of angle $\alpha$ is modeled as charge sheets of charge density $\pm B_{r} \sin \alpha$ at the top and bottom of the PM block, respectively. Where two adjacent PM blocks are mismatched in strength, there is are error charge sheets of 


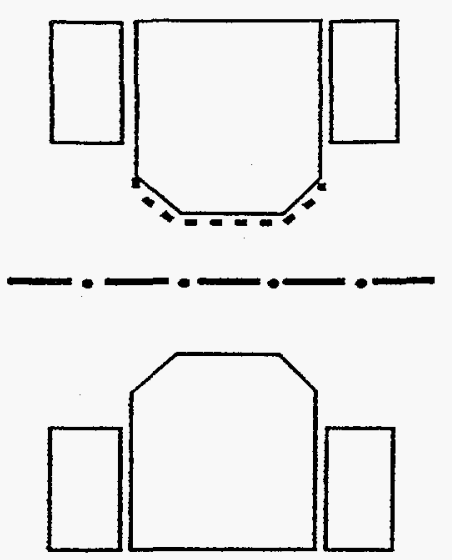

Vertical pole motion
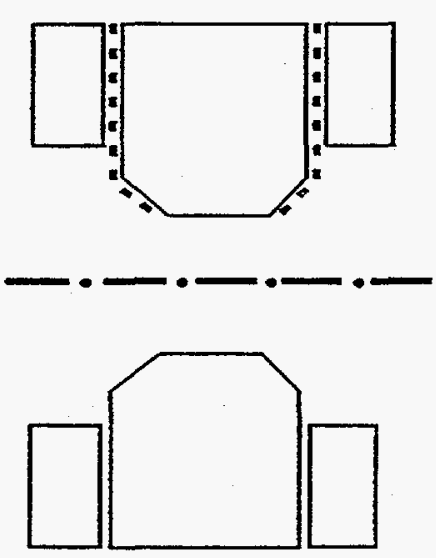

Pole thickness variation

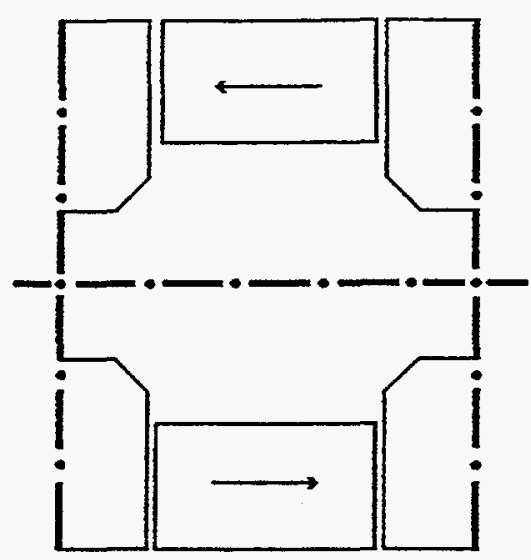

Spacing variation between pole and CSEM

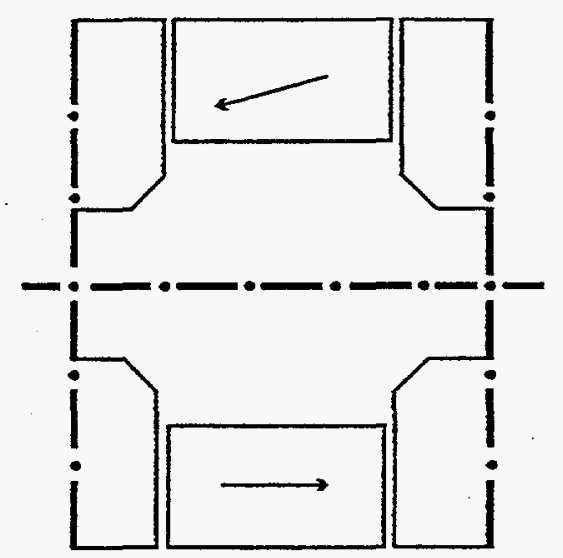

Easy axis misorientation

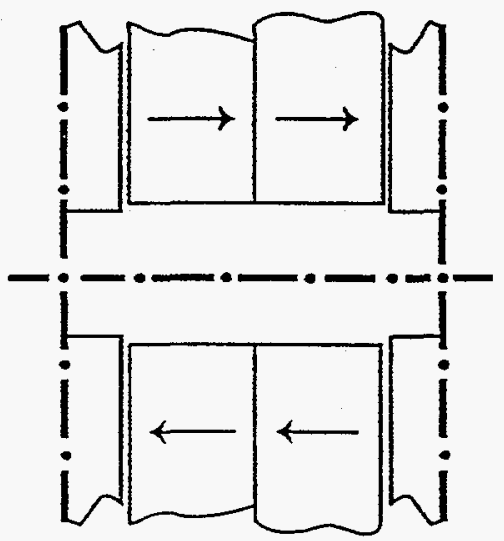

Adjacent block

$\mathrm{Br}$ variation

Figure 7. Various Hybrid ID error sources. 
strength $\pm\left(B_{r_{1}}-B_{r_{2}}\right)$. Chips in PM blocks, oversized PM blocks, and inhomogeneities in PM blocks can also be handled analogously. The relative iomportance of these error sources varies with the geometry, field strengths, etc. Notice that whenever there is an error charge sheet, there is a corresponding error sheet of opposite charge, usually adjacent to a pole, where a feature is or should have been. It should be noted that the only reason there is net flux to the beam is because of three-dimensional effects; if $c_{D_{s}}$ were zero, $Q_{\text {beam }} \longrightarrow 0$ too! Implications for tolerances and PM quality specifications have been discussed at length in the various ALS ID conceptual design reports.

The theory described in this paper is quite general, and is good for all hybrid designs, error analyses, or whenever one wishes to determine out how much of a charge winds up on a given surface.

\section{Acknowledgements}

I am happy to acknowledge Klaus Halbach's original work on the hybrid theory and his guidance in applying the fundamentals to the ALS IDs described herein.

\section{References}

1. K.Halbach, ID Design, Law. Berk. Lab. Rep. V-8811-1.1-16 (1989).

2. K.Halbach, 3-D Hybrid Theory, Law. Berk. Lab. Rep. LSBL-034. (1989).

3. U5.0 Conceptual Design Report, Law. Berk. Lab. Pub-5256 (1989).

4. U8.0 Conceptual Design Report, Law. Berk. Lab. Pub-5276 (1990).

5. W16.0 Conceptual Design Report, Law. Berk. Lab. Pub-5288 (1991).

6. U10.0 Conceptual Design Report, Law. Berk. Lab. Pub-5390 (1994).

7. EW20.0 Conceptual Design Report, Law. Berk. Lab. Pub-5400 (1995).

\section{Appendix A}

The $f=V_{i}\left(\vec{r}_{Q}\right) / V_{0}$. Proof: consider $I \equiv \int\left(V_{i} \vec{B}_{d}-V_{d} \vec{B}_{i}\right) \cdot d \vec{a}$, where $d \vec{a}$ is over all surfaces enclosing the total volume that is not iron. On the reference surface: $V_{d}=0, V_{i}=0$; on surface $1: V_{d}=0, V_{i}=V_{0}$; and at infinity $V \vec{B} \longrightarrow 0$ faster than $a \longrightarrow \infty$. Thus, one expression for $I$ is

$$
I=V_{0} \Phi_{1_{d}}
$$

Alternatively, $I$ can be expressed

$$
\begin{gathered}
I \equiv \int\left(V_{i} \vec{B}_{d}-V_{d} \vec{B}_{i}\right) \cdot d \vec{a}=\int \vec{\nabla} \cdot d\left(V_{i} \vec{B}_{d}-V_{d} \vec{B}_{i}\right) d v= \\
\int\left(V_{i} \vec{\nabla} \cdot \vec{B}_{d}-V_{d} \vec{\nabla} \cdot \vec{B}_{i}+\vec{H}_{d} \cdot \vec{B}_{i}-\vec{H}_{i} \cdot \vec{B}_{d}\right) d v=\int V_{i}(\vec{r}) \rho_{e q}(\vec{r}) d v
\end{gathered}
$$

since $\vec{\nabla} \cdot \vec{B}_{d}=\rho_{e q}, \vec{\nabla} \cdot \vec{B}_{i}=0$, and $\vec{H}_{d} \cdot \vec{B}_{i}=\vec{H}_{i} \cdot \vec{B}_{d}$. The latter can be shown:

$$
\vec{H}_{d} \cdot \vec{B}_{i}=\mu_{0}\left(\vec{H}_{i_{\|}}+\vec{H}_{i_{\perp}}\right) \cdot\left(\mu_{\|} \vec{H}_{\alpha_{\|}}+\mu_{\perp} \vec{H}_{d_{\perp}}\right)=\mu_{0}\left(\mu_{\|} \vec{H}_{i_{1}} \vec{H}_{d_{\|}}+\mu_{\perp} \vec{H}_{i_{\perp}} \vec{H}_{d_{\perp}}\right)=\vec{H}_{i} \cdot \vec{B}_{d}
$$

Equating the two expressions for $I$ gives $\Phi_{1_{d}}=\int V_{i}(\vec{r}) \rho_{e q}(\vec{r}) d v / V_{0}$, which for a point charge $Q$ reduces to $\Phi_{1_{\alpha}}=V_{i}\left(\vec{r}_{Q}\right) Q / V_{0}=f Q$. 